\title{
A User Study of a Computer Retrieval System
}

\begin{abstract}
A study was conducted of the users of the Indiana University PROBE computer retrieval program using the Resources in Education ERIC data base. The critical incident survey identified the PROBE user characteristics, needs, and satisfaction. A typical user profile was determined and overall satisfaction was found to be 79.5 percent. As a result of the study, changes were effected in the retrieval program concerning time lag, price, use of computer fields, and query specificity.
\end{abstract}

A

BETTER UNDERSTANDING OF EDUCATORS and their information needs can be acquired by studying them as users of an information system. Since the purpose of gathering and disseminating information is to satisfy these users, their needs and requirements must be studied to assure the utility of an information system. Both in planning and in evaluating a system, a user's interface is of critical importance.

If inexpensive and rapid retrieval systems are developed and are easily accessible, school teachers and administrators may be encouraged to obtain and use relevant research. Thus, research would be available to the practitioners as well as to the university students and faculty.

When describing the installation of a coordinated information network in the New York State Education Department, Hull and Benson stated:

The improvement of educational pro-

Eva L. Kiewitt is assistant professor, Graduate Library School, Indiana University, Bloomington. cesses and systems depends on the availability of appropriate information for rational decisions. Information must be available in a form which can be understood by a decision-maker, and at a time appropriate to a decision. Many a school superintendent or classroom teacher has acted out of expediency because he was unaware of alternative plans of action. The quality and amount of information available to the decision-maker can be expected to influence the outcome. ${ }^{1}$

Users of a computer retrieval program at Indiana University were studied in the spring of 1973 to determine their characteristics and satisfaction. The data base used in the retrieval program was the Resources in Education (RIE), formerly Research in Education, magnetic tapes from the Educational Resources Information Center (ERIC) system.

ERIC was established by the U.S. Office of Education in 1966 in response to a growing concern for control of proliferating research reports in the field of education. It is now under the sponsorship of the National Institute of Ed- 
ucation (NIE). The national network is composed of sixteen specialized subject centers, or clearinghouses, which select, screen, and abstract educational research reports and disseminate this research by means of microfiche collections and magnetic tapes. The two major ERIC index tools, Resources in Education (RIE) and Current Index to Journals in Education (CIJE), are available both in hard copy and on machine-readable magnetic tapes. $R I E$ was begun in 1968 as a monthly abstract journal with subject, author, and institution indexes to the ERIC research reports.

In order to make information readily available to both practitioners and researchers, many libraries and information centers began to develop local retrieval computer programs. PROBE, a batch-mode retrieval program of the ERIC data base, was developed in 1970 by Ronald Tschudi at Indiana University. The program has a variable-field format which gives it a broad range of capabilities. Details of the program are described in a technical report published in 1972 by Tschudi and Meredith, ${ }^{2}$ and the history of its development was reported in $1973 .^{3}$

The retrieval program was supported by aid from the University, a one-year LSCA grant from HEW awarded through the Indiana State Library, and a $\$ 4.00$ fee per search from the users. With the aid from the grant it was possible to expand service to educators throughout the state of Indiana and accept some out-of-state requests for searches.

\section{USER NeEds ANd STUdies}

After the retrieval system had been in use more than a year, it was decided to evaluate the system by studying the needs and satisfaction of the users. The user "feedback" could help determine changes needed to improve the system and satisfy the users.
The first major evaluation study of information retrieval systems was the Cranfield investigation in England. Four indexing systems were tested and the studies were summarized by Cleverdon in 1962. The major results of the tests were not only the comparison of the indexing systems but methods of evaluation. ${ }^{4}$ Swanson reviewed the Cranfield experiments in 1965, citing some of the major findings. He stated the value of the project but criticized the inaccurate interpretations and generalizations of the Cranfield data. ${ }^{5}$

In discussing evaluation of systems, Cleverdon stated:

In future work in evaluation testing, the users must be included as an integral part of the system and their requirements must be evaluated along with the other system components. ${ }^{6}$

Few systems are viable without "feedback" from the user, since information is stored in anticipation of some user's needing it. After reviewing studies of user needs from 1953 to 1966 , Coover said:

A determination of the needs of users is absolutely essential to the management of an information center. . . . In fact, the value of the Information Center can be known only in terms of its satisfying the users' needs. ${ }^{7}$

There are various techniques of analyzing user needs. One method used frequently in user studies is the critical incident survey, which relates to a particular incident of searching for information. This method was used in Lancaster's evaluation of the MEDLARS Demand Search Service of medical literature from 1966 to 1968 . He studied twenty-one user groups and 607 requests and analyzed 302 actual searches. ${ }^{8}$

In each of the volumes of Annual Review of Information Science and Technology from 1966 to the present, a chapter has been devoted to information needs and uses. A review of these 
studies of user needs and the methods used to determine their requirements showed that the most viable method of studying the PROBE users was the critical incident technique.

\section{Critical Incident SuRvey}

A critical incident survey of PROBE users was conducted in the spring of 1973. As each user requested a computer search by means of mail, telephone, or "walk-in," a search request form was completed, either by the librarian or by the user. The request form included space for basic information about the patron, a description of the problem, and a place for descriptors or other elements to be used in formulating the search query.

The description of the patron's subject of interest was discussed thoroughly with the individual if the request was made in person or by telephone. If the search arrived by mail, the librarian examined the user's statement of the problem or request and chose the terms to be searched by the computer.

Boolean logic was used to combine the selected descriptors (subject headings) to form the computer query, together with the logical connectors "and" (conjunction), "or" (disjunction), and "not" (negation).

Since the PROBE computer program is very flexible, it is possible to search the tapes in many ways. The majority of users have been able to find descriptors listed in the Thesaurus of ERIC Descriptors to fit their interests. ${ }^{9}$ However, if no descriptor is judged to be relevant, a search may be made through other bibliographic fields, such as the title, the author, the identifier (proper nouns, such as Indiana University), or through the abstract field. The abstract may contain words or phrases that are not used as descriptors, and a search through this summary paragraph with the use of natural language terms may locate a relevant article not found by descriptor search.

The user received a complete printout of the first 100 abstracts and up to 700 additional citations, if the inquiry generated that many hits.

The search request forms were sent daily, Monday through Friday, to a keypunch operator, who then submitted them at the Wrubel Computer Center in batch-mode, three times each week.

Following completion of each computer search, either the patron was notified of the receipt of the search or the printout was mailed. A cover letter was attached to each printout asking that the enclosed questionnaire be completed and returned after the user's appraisal of the results.

From the questionnaires the characteristics of the user were obtained: the purpose of the search, satisfaction, and willingness to pay. Information about age, sex, position, level of education, and subject interest area was requested in order to obtain a profile of the users. Satisfaction was inferred from answers to questions concerning relevancy of the output, the worth of nonrelevant matches, the number of documents new to the user, usefulness of the information, and good features and problems of the computer search.

\section{User Characteristics}

The questionnaire, with a cover letter of explanation, was included with the computer output of 200 consecutive searches. Of these 200 questionnaires, 156 (78 percent) were returned. Some of the users requested more than one search during the evaluation period, and, consequently, the number of individual users who returned the questionnaire was 141 .

The majority of users were from college and university campuses. Students and faculty from Indiana University comprised 63.1 percent; from other Indiana universities, 14.9 percent; users 
from Indiana elementary and secondary schools, 12.8 percent; and users from out-of-state locations, 9.2 percent.

The age group of the users ranged from eighteen to twenty-one to fortysix to fifty-five years of age. The largest number of users (sixty-four, or 47.8 percent) were in the twenty-six to thirtyfive age-group category.

The educational levels of the respondents ranged from high school graduates (ten, or 7.1 percent) to those with doctorates (twenty-six, or 18.4 percent). The largest number of users reported the master's degree as their highest academic degree (fifty-six, or 39.7 percent). The majority of the users were male (eighty, or 56.7 percent), with sixty-one, or 43.3 percent, female.

The occupations of the users were quite varied. The primary user was the graduate student (sixty-seven, or $\mathbf{4 7 . 5}$ percent), with college faculty as the second largest group (twenty, or 14.2 percent). The greatest number of users were associated with a college or university campus. This was anticipated since the ERIC data base is primarily oriented toward research; also, the PROBE service and most ERIC collections are located on university campuses. However, twenty-nine (20.6 percent) of the users listed elementary, middle, or high school as their primary institutional association.

Sixty (42.5 percent) of the users indicated that they had conducted research within the past five years; and thirty-eight (26.9 percent) of the users stated that they had published articles or books or presented papers during that period.

The individual respondents were queried concerning their use of ERIC material. Of the users, 107 ( 75.9 percent) indicated they had easy access to ERIC collections, usually at one of the four state universities or private colleges in Indiana. Seventy-two percent of the users stated that they found the use of microfiche acceptable but not preferable. They noted that it is easy to read and store, the necessary data are available on microfiche, and it is less expensive than hard copy.

The PROBE office was interested in learning where users had obtained information concerning the availability of the PROBE computer service. The greatest number (27.2 percent) stated that they had heard of PROBE from a colleague. The three other methods of communication that were most effective were: librarian, 22.8 percent; teacher, 21.7 percent; and brochure or flyer, 10.9 percent.

\section{SATISFACTION OF PROBE UsERS}

The satisfaction of the users was ascertained by requesting information on the purposes of their PROBE searches and asking for the overall usefulness or value of their results.

A number of users indicated that they requested the computer search for more than one reason, as is shown in Table 1.

The users indicated research as the major purpose for a PROBE search (49 percent); of this number, forty-two specifically mentioned that it was for a dissertation. A few others said that they were searching for a dissertation topic.

TABLE 1

Purposes of the Computer Searches

\begin{tabular}{lcc}
\hline \hline \multicolumn{1}{c}{ Purpose of Search } & $\begin{array}{c}\text { Number of } \\
\text { User Purposes }\end{array}$ & Percent \\
\hline $\begin{array}{l}\text { Research project } \\
\text { (Dissertation, } \\
\text { specifically, 42) }\end{array}$ & 101 & 49.0 \\
$\begin{array}{l}\text { Assignments or term paper } \\
\text { Preparation of speech, }\end{array}$ & 41 & 19.9 \\
$\begin{array}{l}\text { report, or article } \\
\text { Curriculum development }\end{array}$ & 21 & 10.2 \\
$\begin{array}{l}\text { Program improvement } \\
\text { To keep abreast in a field }\end{array}$ & 9 & 8.2 \\
Preparation or updating of & 4 & 4.4 \\
$\quad$ course bibliographies & 2 & 1.9 \\
$\begin{array}{l}\text { Browsing } \\
\text { Other }\end{array}$ & 2 & 1.0 \\
Total purposes & 3 & 1.5 \\
\hline
\end{tabular}


The crucial question concerning satisfaction was how the users rated each search as to its usefulness to their study or work. Of the 156 searches conducted for 141 users, their value was indicated in 151 of the responses, as shown in Table 2.

TABLE 2

Usefulness of the Descriptor Searches

\begin{tabular}{lcc}
\hline \hline \multicolumn{1}{c}{ Value of the Search } & $\begin{array}{c}\text { Number of } \\
\text { Searches }\end{array}$ & Percent \\
\hline Very high value & 42 & 27.8 \\
High value & 52 & 34.5 \\
Medium value & 26 & 17.2 \\
Little value & 16 & 10.6 \\
No value & 15 & 9.9 \\
Total & 151 & 100.0 \\
\hline
\end{tabular}

Ninety-four (62.3 percent) of the searches were considered of high or very high value to the users; twenty-six (17.2 percent) of medium value; and thirtyone ( 20.5 percent) of little or no value. Thus, overall, 120 (79.5 percent) considered the searches of value compared with 31 (20.5 percent) who felt that their searches were of little or no value.

The users indicated that they found more relevant reports than nonrelevant in their searches. However, since some nonrelevant material was found, the users were asked to indicate whether they felt it was worthwhile examining this material. Fifty-six percent of them felt that it was worthwhile to examine this additional material.

The time lag from request of the search to the receipt is often very important to users. Thus, it was necessary to determine if the users felt the turnaround time of the PROBE search was adequate for their needs. During the evaluation period the PROBE schedule had been to process the searches three times a week in batch-mode. Unless mailing time was involved, the user usually could obtain a search in two or three days. Eighty-eight percent of the users felt that the length of time from request to receipt of their printout was either fast or reasonable.

A large number of users ( 81.5 percent) indicated that much of the information obtained in the computer searches was new. The novelty ratio was 60.9 percent, which shows that over half of the relevant information obtained was new to the user.

Users were asked to identify both good features and problems with their searches. A larger number of users indicated satisfaction with the computer search than dissatisfaction. The three good features of the computer search most frequently identified were time saving, speed, and comprehensiveness. The two problems listed most often were lack of adequate descriptors and time lag.

Users were also requested to indicate their willingness to pay. Sixty-eight percent of the users were willing to pay from $\$ 5$ to $\$ 10$ per search, and 32 percent stated their willingness to pay more than $\$ 10$ per search. Some were willing to pay as much as $\$ 60$. Thus, the majority were quite willing to pay within the present price range, and approximately one-third were willing to pay from $\$ 10$ to $\$ 60$.

\section{SUMMARY OF USER STUdY}

A profile of the most typical PROBE user, determined from the questionnaires, showed that he was a male graduate student, between the ages of twenty-six and thirty-five, working on research at Indiana University (Bloomington Campus). Other larger user categories were college faculty members, twenty, or 14.2 percent; undergraduate students, sixteen, or 11.3 percent; and administrators, fifteen, or 10.6 percent. Overall satisfaction of the users proved to be high since 79.5 percent of the searches were considered of value.

Because of the user study, the following changes were effected in the retrieval program: the time lag was shortened, 
the price per search was raised slightly (from $\$ 4$ to $\$ 6$ ), more computer fields were used to allow flexibility, and the queries were structured with more specificity.

The number of searches requested has remained stable, the price increase not having altered the demand for such a service. The improvements effected in the program following the evaluation have kept it a popular retrieval system in Indiana and the number of out-ofstate requests has increased steadily. More detailed information regarding the study may be obtained from the author's complete description of the evaluation. ${ }^{10}$

\section{References}

1. W. L. Hull and Gregory Benson, Jr., Installing a Coordinated Information Network in a State Education Agency: A Case Study of the Decision Process in New York. Final Report (Albany, N.Y.: New York State Education Dept., Feb. 1972), p.15. (ERIC, ED 061 428).

2. R. R. Tschudi and J. C. Meredith, The "PROBE" Retrieval Program-A Description (Bloomington: Indiana Univ., Feb. 1972). (ERIC, ED 059596 ).

3. Eva L. Kiewitt, PROBE Computer Search of the ERICtapes (Bloomington, Ind.: Indiana Univ., May 1973). (ERIC, ED 070 050 ).

4. C. W. Cleverdon, Factors Determining the Performance of Indexing Systems (Cranfield, England: College of Aeronautics, 1966 ).

5. D. R. Swanson, "The Evidence Underlying the Cranfield Results," Library Quarterly 35:1-20 (Jan. 1965).

6. C. W. Cleverdon, "Design and Evaluation of Information Systems," Annual Review of Information Science and Technology 6:68 (1971).

7. R. W. Coover, "User Needs and Their Effect on Information Center Administration: A Review, 1953/66," Special Libraries 60: 446-47 (Sept. 1969).

8. F. W. Lancaster, Evaluation of the MEDLARS Demand Search Service (Washington, D.C.: National Library of Medicine, Jan. 1968). (ERIC, ED 022 494).

9. Thesaurus of ERIC Descriptors (New York: CCM Information Corp., 1972).

10. Eva L. Kiewitt, "PROBE: Computer Searches of the ERICTAPES-An Evaluation of a Pilot Study" (Ph.D. dissertation, Indiana Univ., 1973). 